\title{
Halloysite nanotubes: Prospects in electrorheology
}

\author{
N. M. Kuznetsov ${ }^{1 *}$, D. Yu. Stolyarova ${ }^{1,2}$, S. I. Belousov ${ }^{1,3}$, R. A. Kamyshinsky ${ }^{1,4}$, A. S. Orekhov ${ }^{1,5}$, \\ A. L. Vasiliev ${ }^{1,5}$, S. N. Chvalun ${ }^{1,2}$ \\ ${ }^{1}$ National Research Center «Kurchatov Institute», 1, Akademika Kurchatova pl., 123182 Moscow, Russia \\ ${ }^{2}$ Enikolopov Institute of Synthetic Polymer Materials RAS, 70, Profsoyuznaya st., 117393 Moscow, Russia \\ ${ }^{3}$ Karpov Research Institute of Physical Chemistry, 3-1/12, build 6 Obuha lane, 105064 Moscow, Russia \\ ${ }^{4}$ Moscow Institute of Physics and Technology, Institutsky lane 9, Dolgoprudny, 141701 Moscow region, Russia \\ ${ }^{5}$ Federal Research Centre «Crystallography and Photonics» of Russian Academy of Science, 59, Leninskiy prospect, \\ 119333 Moscow, Russia
}

Received 23 March 2018; accepted in revised form 31 May 2018

\begin{abstract}
Electrorheological fluids based on polydimethylsiloxane filled with halloysite nanotubes were studied. The filler structure was characterized by TEM, SEM, and X-ray diffraction. When an electric field is applied to suspensions, their rheological behavior changes - the contribution of the elastic component becomes significant and samples behave like a solid body. The effect of the electric field and filler concentration on the electrorheological behavior was investigated. The influence of water content on the filler structure, as well as on electrorheological and electrophysical properties of suspensions, was considered. Electrorheological fluids filled by halloysite with small water content exhibit slightly higher rheological characteristics under an electric field than dried ones. This study shows the prospects of using halloysite nanotubes as a dispersed phase for electrorheological fluids.
\end{abstract}

Keywords: rheology, electrorheological fluid, halloysite nanotube, electron microscopy, X-ray diffraction

\section{Introduction}

Disperse systems consisting of semiconductor particles in a dielectric medium are able to change their visco-elastic properties under the electric field. Such behavior is called the electrorheological effect, and materials as electrorheological fluids (ERFs) [1]. The fast and reversible response of ERFs to the applied electric field makes it possible to classify them as smart materials [2]. ERFs show their performance in vibration control systems, dampers, sensors, microfluidics and robotics [3-8].

The mechanism of the electrorheological effect is in the polarization of disperse phase particles by an electric field leading to the sample solidification due to chain-like structure formation between electrodes [911]. The electrorheological effect depends on various factors, i.e. particle size, aspect ratio, concentration and the difference in permittivities of medium and particle. If nanofillers of high aspect ratio $\left(l / d \sim 10^{1}-\right.$ $10^{2}$ ) are used, a continuous network are formed at low fillings (less than $10 \mathrm{wt} \%$ ) [12] and, as a consequence, a significant electrorheological effect can be obtained. One of such promising candidates with high aspect ratio is natural nanoclay halloysite [13]. The morphology of halloysite can be various, but more often it is multilayered nanotubes with a cavity inside. The tube is formed by a folded aluminosilicate sheet, with elementary lattice formed by a tetrahedral silica-oxygen and octahedral alumino-hydroxyl layers. These layers are linked by hydrogen bonds. Various molecules can penetrate into the cavity, for example, a dispersion medium in the case of ERF. Two types of halloysite are known, with interplanar distances of 10 and $7 \AA$. The first one with increased

*Corresponding author, e-mail: kyz993@yandex.ru

(C) BME-PT 
interplanar distance is the hydrated form and the second can be obtained by heating halloysite above $50^{\circ} \mathrm{C}$. Thus, nanotubes easily lose a significant amount of water and pass from one form to another [14]. In the last decade several papers were reported about the application of layered aluminosilicates and polymer composites based on them as fillers for electrorheological fluids [15-19]. At the same time, the number of such articles is not significant, and only few of them are devoted to systems with halloysite [20-22]. In these papers, the electrorheological effect of halloysite suspension in silicon oil and its PANI-based and PPy-based composites was demonstrated. At $5 \mathrm{wt} \%$ filler content in halloysite suspension the yield stress was about $7.5 \mathrm{~Pa}$ at an electric field of $2 \mathrm{kV} / \mathrm{mm}$ [21]. It can be noted that composites show more significant electrorheological effect. PANI-based composite (halloysite content about $91 \mathrm{wt} \%$ ) [20], the yield stress was about $60 \mathrm{~Pa}$ at the same field for ERF filled by $15 \mathrm{wt} \%$. The authors note the classical quadratic dependence of the yield stress on electric field. Nevertheless, PPy-based composite [22] showed the yield stress 22.6 Pa at $10 \mathrm{vol} \%$ and electric field $2 \mathrm{kV} / \mathrm{mm}$. The dependence of the yield stress on electric field in this case was not quadratic and changes from 1.5 to 1 at electric field value $2.5 \mathrm{kV} / \mathrm{mm}$. However, the influence of halloysite concentration, the tubular filler shape and dielectric characteristics on electrorheological effect was not considered. The aim of this work was to study the characteristics of ERFs filled by halloysite nanotubes at different concentrations up to $8 \mathrm{wt} \%$ with detailed characterization of filler shape and dielectric properties.

\section{Materials and methods}

Polydimethylsiloxane (PMS-100 brand, GOST 13032-77, Penta Junior LLC, Russia) was used as the dispersion medium. According to GPC data an average PMS-100 molecular weight is $M_{\mathrm{w}}=$ $10700 \mathrm{Da}, P D I=1.8$. The dynamic viscosity is $0.105 \mathrm{~Pa} \cdot \mathrm{s}$. Halloysite nanotubes were obtained from Sigma-Aldrich, USA. The tube length is $1-3 \mu \mathrm{m}$, and the diameter is $30-70 \mathrm{~nm}$. Two kinds of the filler were used: the pristine filler and a dried one, dried under vacuum at $60^{\circ} \mathrm{C}$ for 24 hours and then at $80^{\circ} \mathrm{C}$ for 2 hours. The difference in water content for pristine and dried halloysite was $2.2 \mathrm{wt} \%$ measured by Karl Fischer coulometric titration method. This data correlate well with previously obtained value by TGA analysis [23]. The suspensions with 1, 2, 4, $8 \mathrm{wt} \%$ halloysite content have been prepared by dispersing nanoclay in polydimethylsiloxane oil during 48 hours by means of a magnetic stirrer and following treatment in an Elmasonic ultrasonic bath $\mathrm{S}$ $40 \mathrm{~N}, 340 \mathrm{~W}, 37 \mathrm{kc} / \mathrm{s}, 20 \mathrm{~min}$ before measurements. High resolution transmission electron microscopy (HR TEM) images were obtained in a Titan Krios 60-300 (FEI, USA) cryoelectron microscope, equipped with a high sensitive direct electron detector Falcon II (FEI, USA) and Cs image corrector (CEOS, Germany), at an accelerating voltage of $300 \mathrm{kV}$. The sample was deposited on the lacey carbon EM grid. The study was carried out at liquid nitrogen temperature, which allowed to minimize the radiation damages and amorfization of a sample during the exposure. Scanning electron microscopy (SEM) data were obtained with Versa 3D DualBeam (FEI, USA) in secondary electron (SE) mode at accelerating voltage of $5 \mathrm{kV}$. For image processing and size determination of halloysite nanotubes Fiji software [24] was used.

$\mathrm{X}$-ray diffraction patterns were recorded at DIKSI station of Kurchatov synchrotron radiation source, the used wavelength was $\lambda=1.625 \AA$, and the $2 \mathrm{D}$ detector-Dectris Pilatus $31 \mathrm{M}$. Filler powders and suspension samples were examined in X-ray quartz capillaries Hilgenberg, diameter $2.0 \mathrm{~mm}$, the wall thickness of capillary was 10 microns. The sample-detector distance was evacuated. Silver behenate $\left(d_{001}=\right.$ $58.38 \AA$ ) was used for distance calibration.

Novocontrol Alpha-A impedance analyzer with a ZGS Alpha Active Sample Cell with gold-plated disc electrodes $20 \mathrm{~mm}$ in diameter was used for the dielectric studies. The frequency of measurements was changed in the range $1-10^{6} \mathrm{~Hz}$, the temperature was $20^{\circ} \mathrm{C}$, the voltage applied to the electrodes was $1 \mathrm{~V}$. Electrorheological properties were studied on Anton Paar Physica MCR 501 rheometer, its measuring system consists of two coaxial cylinders (measuring cell CC-27E). The potential was supplied from the constant high voltage source FuG HCP 14 - 12500 MOD to the inner cylinder. The cell volume is $20 \mathrm{~mL}$, the gap is $1 \mathrm{~mm}$, the electric field strength was varied in the range $0-7 \mathrm{kV} / \mathrm{mm}$, with a step of $1 \mathrm{kV} / \mathrm{mm}$, and the temperature of $20^{\circ} \mathrm{C}$. In all measurements the leakage current density did not exceed $30.0 \mu \mathrm{A} / \mathrm{cm}^{2}$ at an electric field of $7 \mathrm{kV} / \mathrm{mm}$. Measurements were carried out in a control shear stress (CSS) mode. To determine the loss, storage moduli and the complex 
viscosity the dynamic measurements were carried out at the strain amplitude value of $0.1 \%$ in the linear range of viscoelasticity.

\section{Results and discussion}

The morphology of halloysite particles essentially depends on natural crystallization conditions and geological occurrences [14]. Therefore, to identify the particles type, halloysite samples were investigated by TEM and SEM. In Figure 1 polydisperse halloysite powder is shown. Randomly oriented clay nanotubes with the length of $50 \mathrm{~nm}-2 \mu \mathrm{m}$ and the diameter of 20-300 nm can be seen (Figure 1a). HR TEM images (Figure 1b-1d) show hollow halloysite nanotubes, different in length, diameter and width of walls. The halloysite walls consist of 11-20 layers with interplanar distance of $0.81 \mathrm{~nm}$ (Figure 1b). Figure 1c shows a halloysite particle with the walls consisted of roughly 23 layers, oriented along the electron beam.

The rheological behavior of colloid systems is determined by the interaction of particles with each other and the dispersion medium. The presence of water adsorbed on the filler surface can significantly affect not only the dispersion process, but its electrophysical properties as well. Thus, X-ray diffraction patterns of powders were recorded to assess the structural changes occurring during the filler drying, (Figure 2). The X-ray diffraction curves of halloysite contain a characteristic smectic peak $(h k)$ at $q \approx$ $14.1 \mathrm{~nm}^{-1}$. This reflection corresponds to the ordered structure of the nanoclay sheet rolled into the tube.

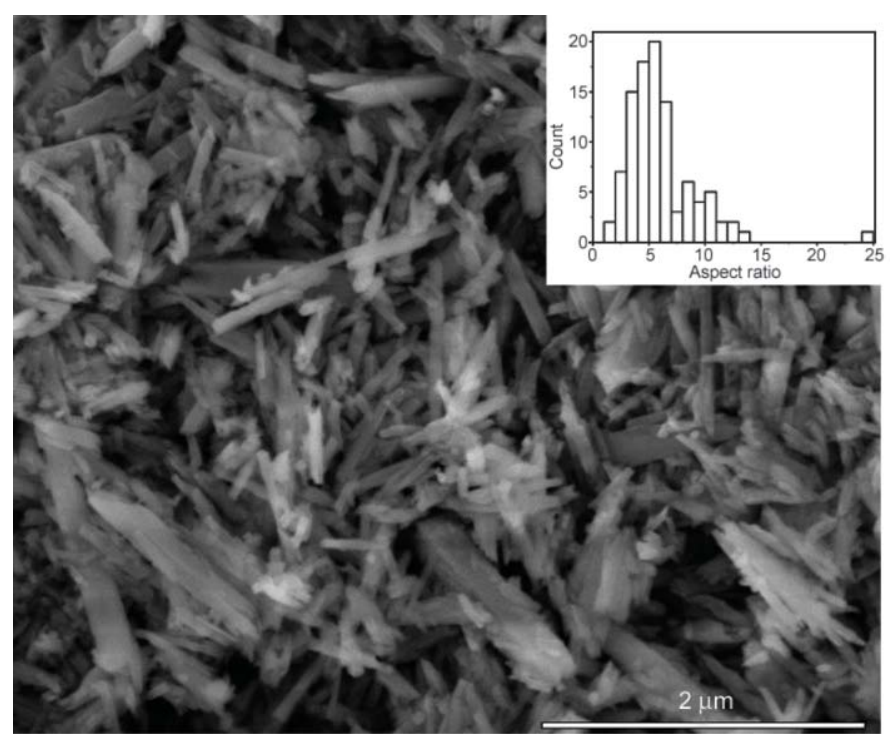

a)

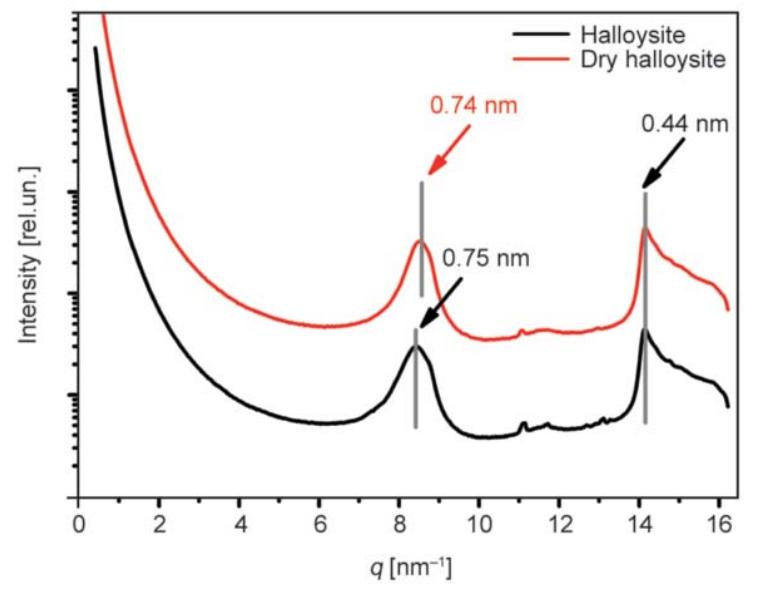

Figure 2. X-ray diffraction patterns of halloysite powder: pristine (-) and dried (-).

Small-angle $00 l$ peak is in the region of $q \approx 8.4 \mathrm{~nm}^{-1}$ and corresponds to interplanar distances in a multilayer tube. The maximum distance $0.74 \mathrm{~nm}$ was detected for the initial filler form, and the same distance was determined to the dehydrated form, thus, in our study, we used meta-halloysite or halloysite $7 \AA$ [14]. The peaks positions of the pristine and dried forms practically coincide. Thus, during the powder dehydration, only water adsorbed on the surface is removed. The presence of water does not change halloysite structure, but it may affect the dielectric characteristics of samples as was mentioned above.

Dielectric permittivity and conductivity values for the dispersion medium and fillers at the frequency of $1 \mathrm{~Hz}$ are presented in Table 1. Polydimethylsiloxane is a dielectric liquid with low values of permittivity and conductivity. Dielectric permittivity of both fillers

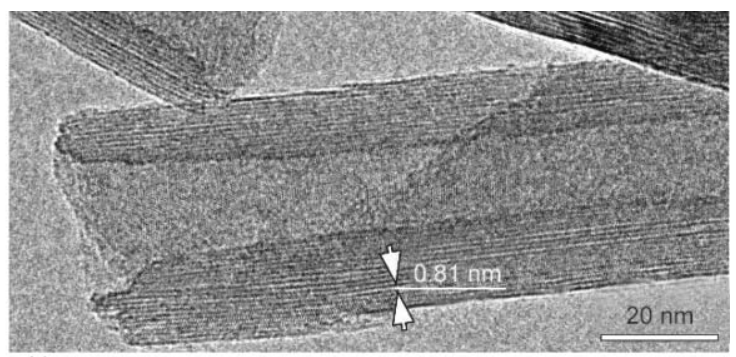

b)

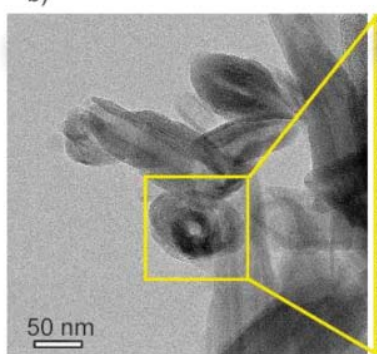

c)

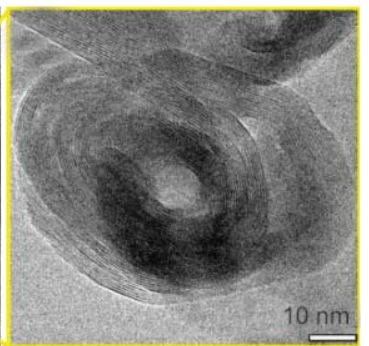

d)

Figure 1. Secondary electron SEM (a) and high resolution TEM images of halloysite particles (b-d). 
Table 1. The values of permittivity and conductivity at a field frequency of $1 \mathrm{~Hz}$.

\begin{tabular}{|l|l|l|}
\hline \multicolumn{1}{|c|}{ Sample } & $\boldsymbol{\varepsilon}^{\prime}$ & $\begin{array}{c}\boldsymbol{\sigma}^{\prime} \\
{[\mathbf{S} / \mathbf{c m}]}\end{array}$ \\
\hline Polydimethylsiloxane & 2.51 & $1.42 \cdot 10^{-14}$ \\
\hline Halloysite & $1.66 \cdot 10^{4}$ & $1.19 \cdot 10^{-8}$ \\
\hline Dry halloysite & $2.04 \cdot 10^{4}$ & $9.50 \cdot 10^{-9}$ \\
\hline
\end{tabular}

is higher in 4 orders of magnitude, compared with the dispersion medium. Conductivity values are higher by 5- 6 orders of magnitude, also. It should be noted that dielectric permittivity and conductivity of both pristine and dried forms are practically the same. Comparing the dielectric properties of halloysite with various modified montmorillonites, aluminosilicates with another layered morphology [25-27], it can be seen that the dielectric constant value of halloysite is only by order of magnitude lower than for the sodium form of montmorillonite $\left(\varepsilon^{\prime}=8.09 \cdot 10^{5}\right)$ and the conductivity is lower in more than 50 times $\left(\sigma^{\prime}=\right.$ $\left.6.10 \cdot 10^{-7} \mathrm{~S} / \mathrm{cm}\right)$, respectively. As we show below, such difference open the opportunity to use halloysite as filler for ERFs at higher electric field compared to the sodium form of montmorillonite [26].

Flow and viscosity curves at various concentrations for samples filled with pristine and dried halloysite were measured. Figure 3 shows the dependencies of shear stress and dynamic viscosity on the shear rate for suspensions of various concentrations filled with the pristine halloysite compared to pure polydimethylsiloxane. Shear stress values increase in filled systems and the yield stress appears at $4 \mathrm{wt} \%$ filler content. The yield stress indicates the percolation network formation of interacting particles. The percolation network becomes stronger with filler concentration and the yield stress value increases (see inset

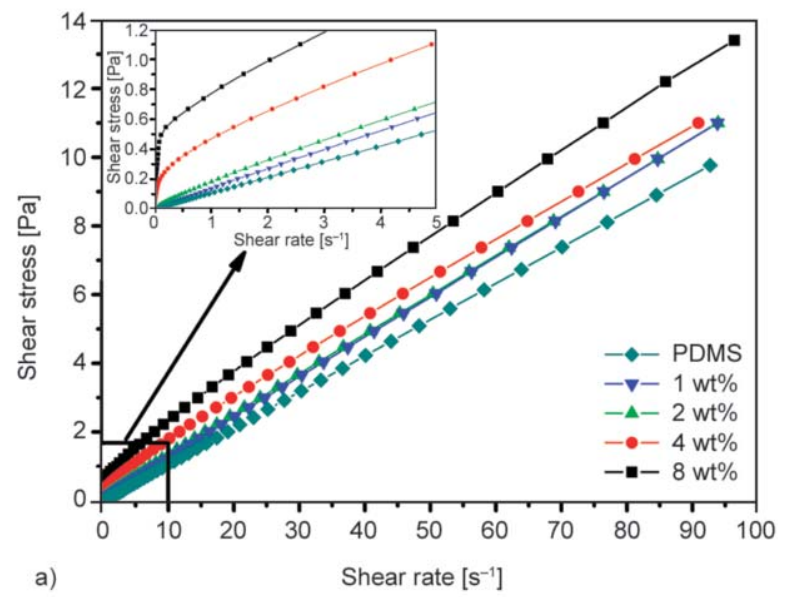

Figure 3a). It is important to note that the yield stress does not appear in montmorillonite suspensions up to $8 \mathrm{wt} \%$ [26]. A possible explanation of such behavior is a better dispersion of halloysite clay tubes in a polydimethylsiloxane medium compared to montmorillonite plates that tend to form tactoids.

Polydimethylsiloxane shows constant viscosity in the whole investigated shear rate range (Newtonian liquid). In halloysite suspensions, viscosity values decrease with shear rate and reach the plateau at high values, exhibiting Newtonian behavior (Figure 3b). Such effect confirms the formation of a continuous network in suspensions with 4 and $8 \mathrm{wt} \%$ filler contents and the elastic component in suspensions with a lower concentration, due to the presence of a weak, fragile structure. It should also be noted the increase in dynamic viscosity values for suspensions in comparison with polydimethylsiloxane.

Frequency tests with strain value of $0.1 \%$ were performed to confirm the obtained results and separate the elastic component in the systems (Figure 4). Modulus values increase with frequency for all samples. Polydimethylsiloxane, as was mentioned earlier, is a Newtonian fluid - there is no storage modulus on frequency dependences. The suspension behavior has not significantly changed by adding $1 \mathrm{wt} \%$ of the filler, there is also just loss modulus detected. However, when the halloysite concentration increases, the storage modulus appears. At $4 \mathrm{wt} \%$ filler content moduli intersect and the crossover point is observed. The storage modulus exceeds the loss modulus at low frequencies $\left(\sim 0.2 \mathrm{~s}^{-1}\right)$. With further concentration increasing the crossover point shifts toward higher frequencies, which indicates the filler network strengthening. Furthermore, a slight increase in moduli values with filler concentration may be noted.

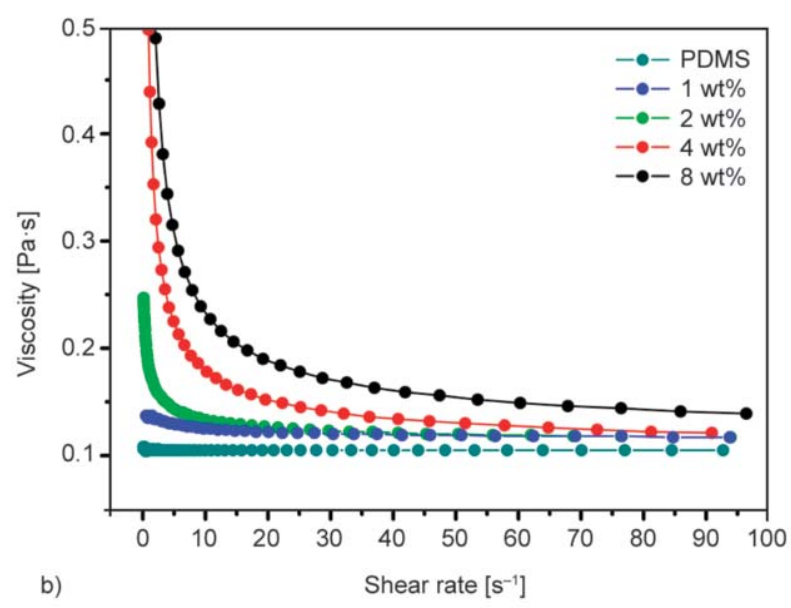

Figure 3. Flow (a) and viscosity (b) curves of halloysite suspensions and polydimethylsiloxane. 


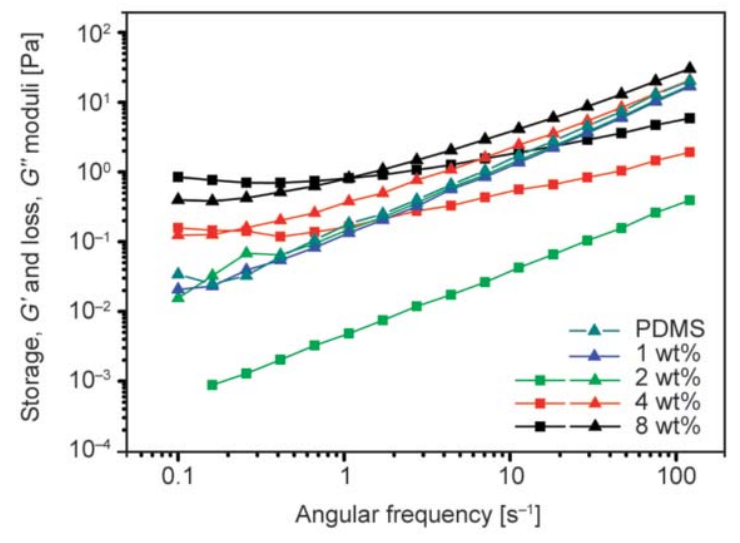

Figure 4. Storage $G^{\prime}(\boldsymbol{\square})$ and loss $G^{\prime \prime}(\boldsymbol{\Delta})$ moduli of halloysite suspensions and polydimethylsiloxane.

The suspensions behavior dramatically changes under electric field. The yield stress appears on flow curves for suspensions with a low filler content and their values increase for all samples with electric field. Flow and viscosity curves of $2 \mathrm{wt} \%$ halloysite suspensions at several electric fields are shown in Figure 5, as an example. There is a significant increase in the viscosity of samples in electric field. The shear rate dependence has a descending character, followed by reaching a plateau at high values. The destruction of formed network is clearly seen from the flow curve at electric field strength of $3 \mathrm{kV} / \mathrm{mm}$ (Figure $5 \mathrm{a}$ ). The slope of flow curve changes at shear rate of $35 \mathrm{~s}^{-1}$, and viscosity reaches a constant value (Figure $5 b$ ).

Flow curves of samples filled by pristine and dried halloysite in concentration range 1, 2, 4 and $8 \mathrm{wt} \%$ at various electric fields were obtained to assess the effect of water on the electrorheological behavior. Dynamic yield stress dependencies on electric field intensity and filler concentration are plotted in Figure 6. A fairly wide spread of yield stresses can be

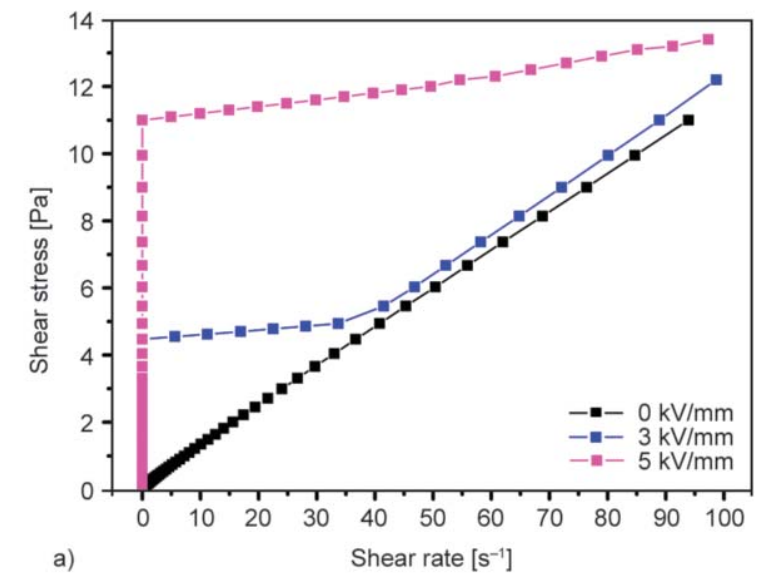

related to the high polydispersity of the filler. Water content does not significantly affect the electrorheological effect at low filler concentrations ( 1 and $2 \mathrm{wt} \%$ ), see Figure 6a, 6b. Such effect is probably associated with low number of columnar structures formed in electric field and with insignificant difference in the dielectric characteristics of fillers. Differences in the electrorheological behavior of pristine and dried halloysite become noticeable at a high filler concentration. It can be clearly seen in dependences for 4 and $8 \mathrm{wt} \%$ suspensions (Figure $6 \mathrm{c}, 6 \mathrm{~d}$ ). The pristine filler form shows slightly higher yield stress values than the dried one. Nevertheless, one can single out the tendency to increase in yield stress values with concentration and electric field increasing in the graph. Thus, water has a weak influence on the electrorheological effect. Values of yield stress for halloysite are lower than for organomodified montmorillonites, for example, the $8 \mathrm{wt} \%$ pristine halloysite suspension reveals yield stress of $51 \mathrm{~Pa}$ at $7 \mathrm{kV} / \mathrm{mm}$, meantime for Cloisite 30B and Nanofil SE 3010 yield stress is 90 and $57 \mathrm{~Pa}$ at the same conditions, respectively [26]. Such difference may be explained by formation of more strong chain-like structures from interacted plates rather than tubes, due to larger overlapping area in former case.

For a more detailed analysis of the ERFs behavior, frequency dependences of storage and loss moduli under the electric field were measured. The linear range of visco-elastic properties can vary when the electric field is applied to the sample. Therefore, amplitude tests were preliminarily carried out at a given angular frequency of $10 \mathrm{~s}^{-1}$ (Figure 7) to select the strain value during the frequency tests. Obtained dependences show a significant decrease in the linear range of visco-elastic properties with increasing

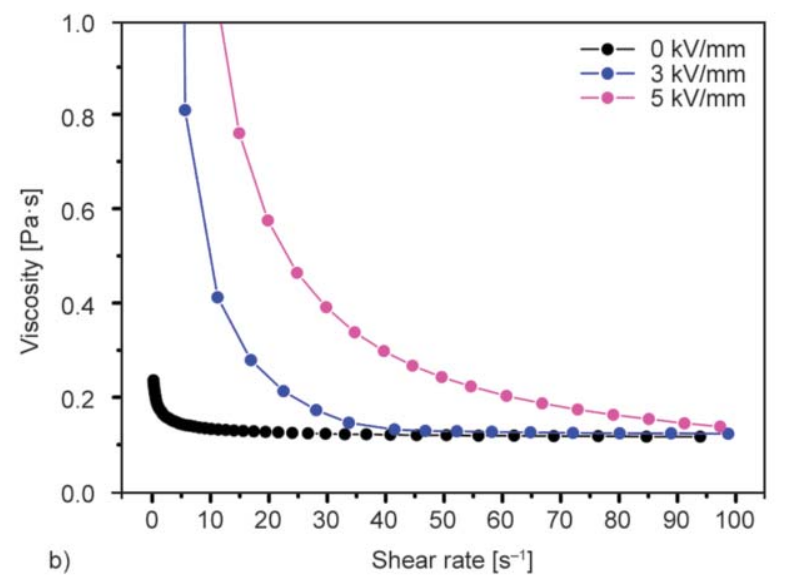

Figure 5. Flow (a) and viscosity (b) curves of $2 \mathrm{wt} \%$ halloysite suspensions at various electric fields. 


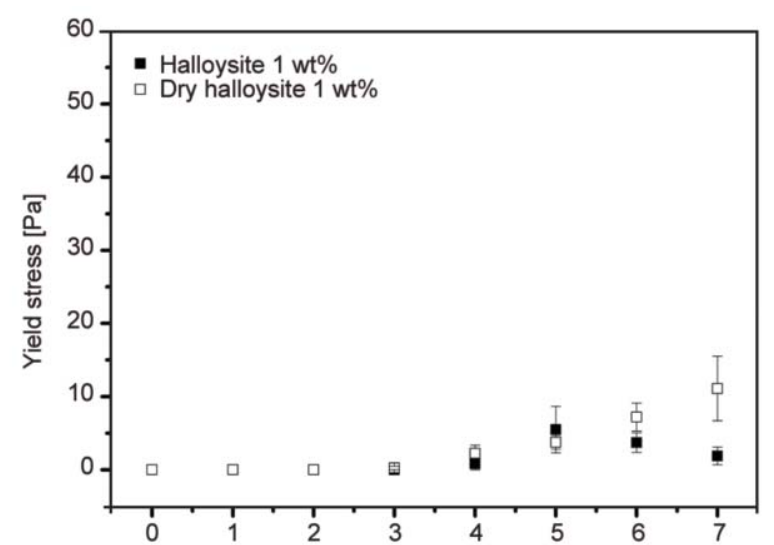

a)

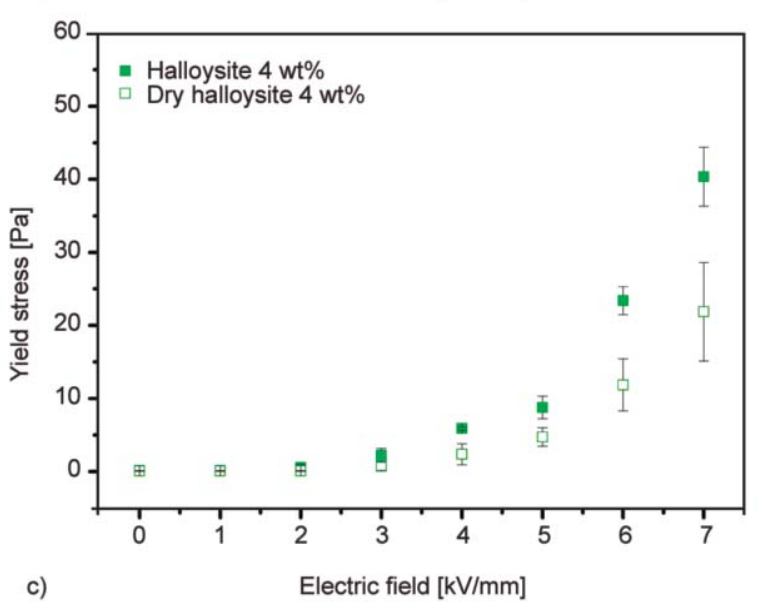

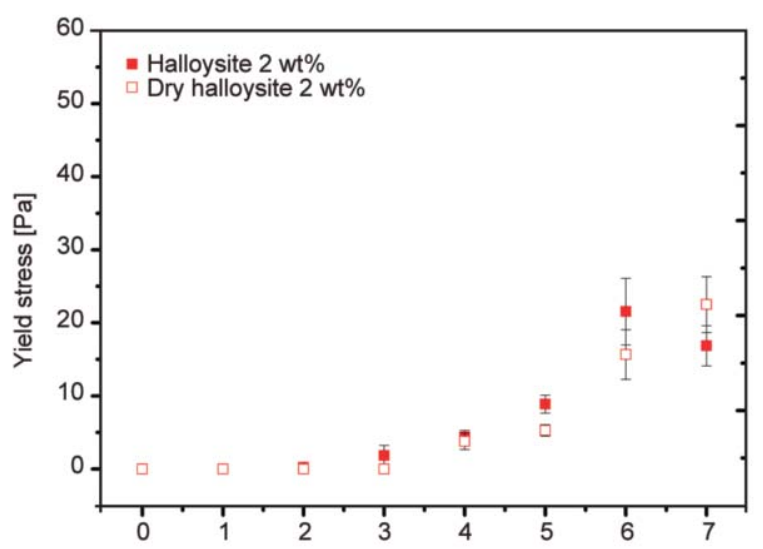

b)

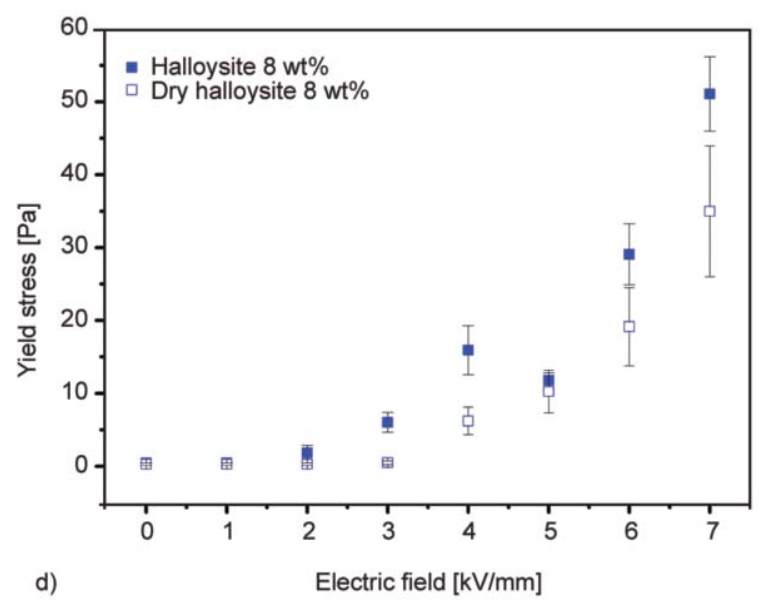

Figure 6. Dynamic yield stress dependence on electric field of halloysite and dry halloysite suspensions at $1 \mathrm{wt} \%$ (a), $2 \mathrm{wt} \%$ (b), $4 \mathrm{wt} \%$ (c) and $8 \mathrm{wt} \%$ (d) filler content.

electric field higher than $3 \mathrm{kV} / \mathrm{mm}$. However, the used strain $(0.1 \%)$ is in the linear region for all studied fields. Figure 8 shows frequency dependences of storage, loss moduli and complex viscosity of $8 \mathrm{wt} \%$ suspensions with pristine and dried halloysite as an example. The intersection of moduli is observed, and the crossover point shifts toward higher frequencies with electric field. The storage modulus exceeds loss modulus over the whole investigated frequency

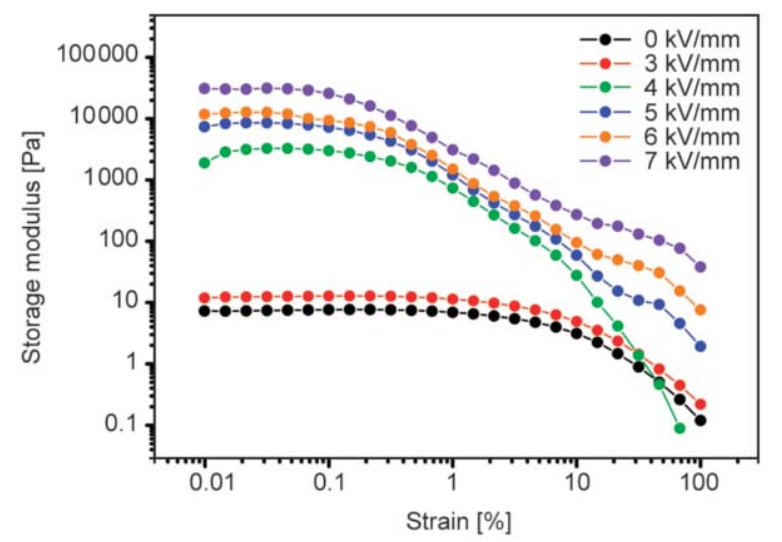

Figure 7. Storage modulus as a function of strain at frequency $10 \mathrm{~s}^{-1}$ of $8 \mathrm{wt} \%$ halloysite suspension. range at electric field higher than $4 \mathrm{kV} / \mathrm{mm}$. Values of storage modulus at electric field $7 \mathrm{kV} / \mathrm{mm}$ reach 20 and $18 \mathrm{kPa}$ for pristine (Figure 8a) and dried (Figure 8c) filler forms, respectively. Complex viscosity for both pristine (Figure 8b) and dried (Figure 8d) filler forms manifests a linear descending character with frequency and even goes to the plateau at low electric fields. Values of complex viscosity increase with electrical potential as well. It is also interesting to note that the obtained values of moduli exceed almost twice the values obtained for systems filled with various montmorillonites. For organomodified montmorillonites the storage modulus value does not exceed $10 \mathrm{kPa}$ at $7 \mathrm{kV} / \mathrm{mm}$ [26].

\section{Conclusions}

Rheological characteristics of low-filled halloysite suspensions in polydimethylsiloxane were studied. Halloysite forms a percolation network even at $4 \mathrm{wt} \%$ content in the media. Suspensions of halloysite in polydimethylsiloxane reveal electrorheological behavior. Values of yield stress increase with electric field and filler concentration. The low water 

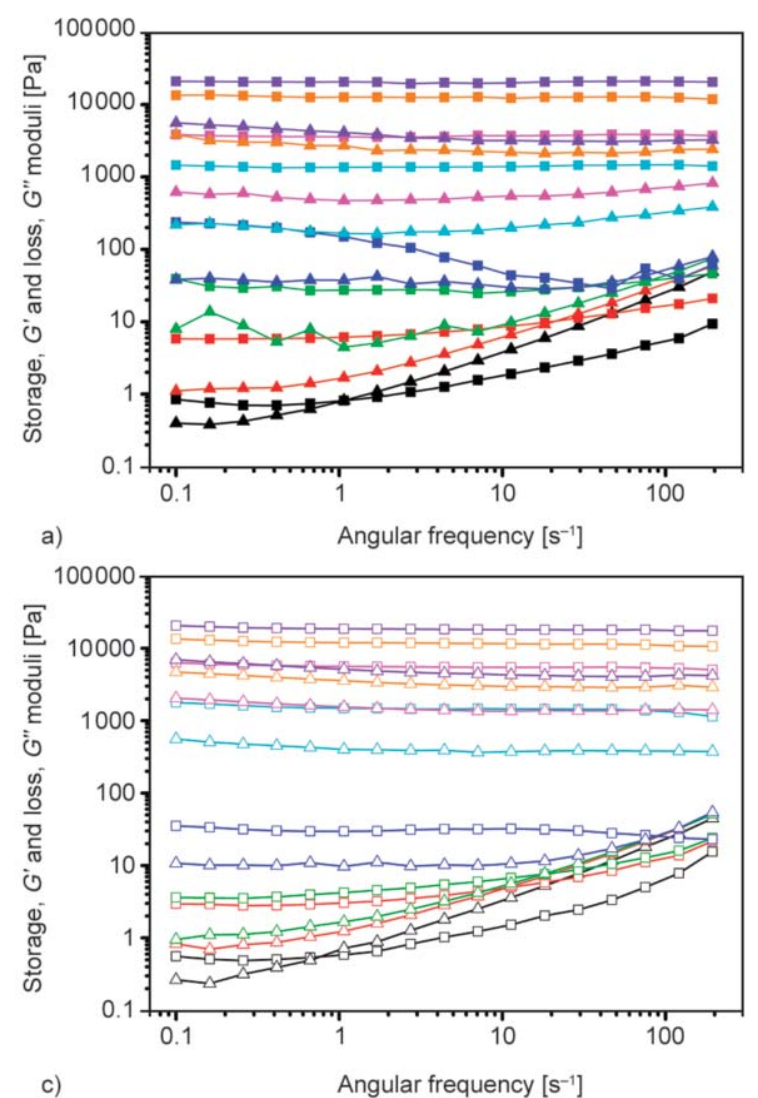
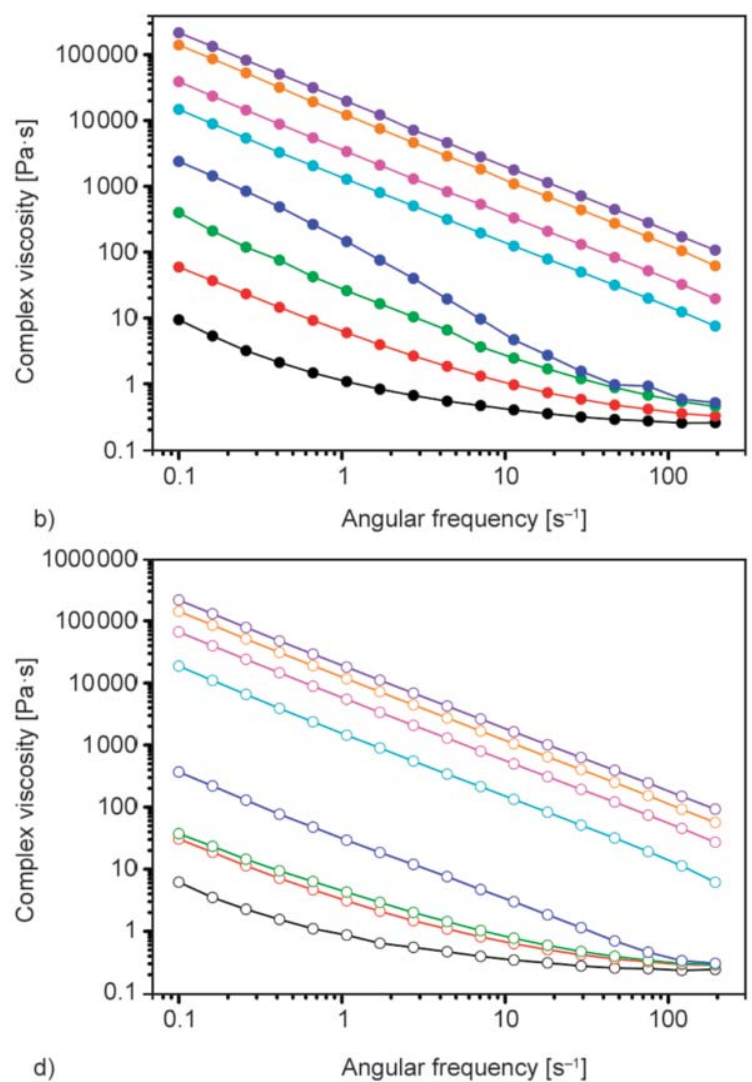

Figure 8. Storage $G^{\prime}(-\boldsymbol{\square}-)$ and loss $G^{\prime \prime}(-\boldsymbol{\Delta}-)$ moduli and complex viscosity $\eta^{*}(-\bullet-)$ of $8 \mathrm{wt} \%$ suspensions halloysite (a, b) and dry halloysite (c, d) at electric field $0 \mathrm{kV} / \mathrm{mm}(-), 1 \mathrm{kV} / \mathrm{mm}(-), 2 \mathrm{kV} / \mathrm{mm}(-), 3 \mathrm{kV} / \mathrm{mm}(-)$, $4 \mathrm{kV} / \mathrm{mm}(-), 5 \mathrm{kV} / \mathrm{mm}(-), 6 \mathrm{kV} / \mathrm{mm}(-)$ and $7 \mathrm{kV} / \mathrm{mm}(-)$.

content does not change filler structure and does not influence the conductivity and permittivity, but resulting in slightly higher yield stresses compared to the dried samples under electric field. The high polydispersity of the filler confirmed by SEM data leads to a significant spread of yield stress values. Frequency dependences of the storage and loss moduli can be understood by continuous network of an interacting particles formation resulting in the increase an elastic contribution with electric field. The research demonstrates the promise of using halloysite nanotubes as filler for electrorheological fluids along with other aluminosilicates.

\section{Acknowledgements}

The authors acknowledge support from the resource center of organic and hybrid materials 'Polymer', the resource center of electrophysical methods 'Electrophysics' and the resource center of probe and electron microscopy 'Nanozond' of the National Research Center 'Kurchatov Institute' for the possibility of rheological, dielectric and electron microscopy studies. And also Kurchatov complex of Synchrotron-Neutron research for the possibility of X-ray structural studies.
The work was partially funded by the Russian Foundation for Basic Research, projects 15-03-01121 A, 18-03-00078 A and 18-33-01123 mol_a.

\section{References}

[1] Winslow W. M.: Induced fibration of suspensions. Journal of Applied Physics, 20, 1137-1140 (1949). https://doi.org/10.1063/1.1698285

[2] Wei Z. G., Sandström R., Miyazaki S.: Shape-memory materials and hybrid composites for smart systems: Part I Shape-memory materials. Journal of Materials Science, 33, 3743-3762 (1998). https://doi.org/10.1023/A:1004692329247

[3] Stanway R., Sproston J. L., El-Wahed A. K.: Applications of electro-rheological fluids in vibration control: A survey. Smart Materials and Structures, 5, 464-482 (1996). https://doi.org/10.1088/0964-1726/5/4/011

[4] Liu Y., Davidson R., Taylor P.: Investigation of the touch sensitivity of ER fluid based tactile display. in 'SPIE Smart Structures and Materials 2005, San Diego, USA' Vol 5764, 92-99 (2005). https://doi.org/10.1117/12.598713 
[5] Hwang Y-H., Kang S-R., Cha S-W., Choi S-B.: An electrorheological spherical joint actuator for a haptic master with application to robot-assisted cutting surgery. Sensors and Actuators A: Physical, 249, 163-171 (2016). https://doi.org/10.1016/j.sna.2016.08.033

[6] Wang L., Gong X., Wen W.: Electrorheological fluid and its applications in microfluidics. Microfluidics, 304, 91-115 (2011).

https://doi.org/10.1007/128_2011_148

[7] Chiolerio A., Quadrelli M. B.: Smart fluid systems: The advent of autonomous liquid robotics. Advanced Science, 4, 1700036/1-1700036/18 (2017).

https://doi.org/10.1002/advs.201700036

[8] Hines L., Petersen K., Lum G. Z., Sitti M.: Soft actuators for small scale robotics. Advanced Materials, 29, 1603483/1-1603483/43 (2017). https://doi.org/10.1002/adma.201603483

[9] Chen T. Y., Briscoe B. J., Luckham P. F.: Microstructural studies of electro-rheological fluids under shear. Journal of the Chemical Society, Faraday Transactions, 91, 1787-1794 (1995). https://doi.org/10.1039/FT9959101787

[10] Bossis G., Métayer C., Zubarev A.: Analysis of chaining structures in colloidal suspensions subjected to an electric field. Physical Review E, 76, 041401/1041401/12 (2007).

https://doi.org/10.1103/PhysRevE.76.041401

[11] Liu Y. D., Choi H. J.: Electrorheological fluids: Smart soft matter and characteristics. Soft Matter, 8, 1196111978 (2012).

https://doi.org/10.1039/c2sm26179k

[12] Okada A., Usuki A.: Twenty years of polymer-clay nanocomposites. Macromolecular Materials and Engineering, 291, 1449-1476 (2006).

https://doi.org/10.1002/mame.200600260

[13] Floody M. C., Theng B. K. G., Reyes P., Mora M. L.: Natural nanoclays: applications and future trends - A Chilean perspective. Clay Minerals, 44, 161-176 (2009). https://doi.org/10.1180/claymin.2009.044.2.161

[14] Joussein E., Petit S., Churchman J., Theng B., Righi D., Delvaux B.: Halloysite clay minerals - A review. Clay Minerals, 40, 383-426 (2005). https://doi.org/10.1180/0009855054040180

[15] Xiang L., Zhao X.: Preparation of montmorillonite/titania nanocomposite and enhanced electrorheological activity. Journal of Colloid and Interface Science, 296, 131-140 (2006). https://doi.org/10.1016/j.jcis.2005.08.059

[16] Erol O., Unal H. I., Sari B.: Synthesis, electrorheology, and creep behaviors of in situ intercalated polyindole/ organo-montmorillonite conducting nanocomposite. Polymer Composites, 31, 471-481 (2010).

https://doi.org/10.1002/pc.20827

[17] Guzel S., Erol O., Unal H. I.: Polyindene/organo-montmorillonite conducting nanocomposites. II. Electrorheological properties. Journal of Applied Polymer Science, 124, 4935-4944 (2012).

https://doi.org/10.1002/app.35594
[18] Wang B., Liu C., Yin Y., Tian X., Yu S., Chen K., Liu P., Liang B.: The electrorheological properties of polyaniline nanofiber/kaolinite hybrid nanocomposite. Journal of Applied Polymer Science, 130, 1104-1113 (2013). https://doi.org/10.1002/app.39262

[19] Geist M. F., Boussois K., Smith A., Peyratout C. S., Kurth D. G.: Nanocomposites derived from montmorillonite and metallosupramolecular polyelectrolytes: Modular compounds for electrorheological fluids. Langmuir, 29, 1743-1747 (2013). https://doi.org/10.1021/la304657p

[20] Zhang W. L., Choi H. J.: Fabrication of semiconducting polyaniline-wrapped halloysite nanotube composite and its electrorheology. Colloid and Polymer Science, 290, 1743-1748 (2012). https://doi.org/10.1007/s00396-012-2719-6

[21] Rozynek Z., Zacher T., Janek M., Čaplovičová M., Fossum J. O.: Electric-field-induced structuring and rheological properties of kaolinite and halloysite. Applied Clay Science, 77-78, 1-9 (2013). https://doi.org/10.1016/j.clay.2013.03.014

[22] Jang D. S., Zhang W. L., Choi H. J.: Polypyrrolewrapped halloysite nanocomposite and its rheological response under electric fields. Journal of Materials Science, 49, 7309-7316 (2014).

https://doi.org/10.1007/s10853-014-8443-5

[23] Polanský R., Kadlec P., Kolská Z., Švorčík V.: Influence of dehydration on the dielectric and structural properties of organically modified montmorillonite and halloysite nanotubes. Applied Clay Science, 147, 1927 (2017).

https://doi.org/10.1016/j.clay.2017.07.027

[24] Schindelin J., Arganda-Carreras I., Frise E., Kaynig V., Longair M., Pietzsch T., Preibisch S., Rueden C., Saalfeld S., Schmid B., Tinevez J-Y., White D. J., Hartenstein V., Eliceiri K., Tomancak P., Cardona A.: Fiji: an open-source platform for biological-image analysis. Nature methods, 9, 676-682 (2012).

https://doi.org/10.1038/nmeth.2019

[25] Giannelis E. P.: A new strategy for synthesizing polymer-ceramic nanocomposites. The Journal of The Minerals, Metals and Materials Society, 44, 28-30 (1992). https://doi.org/10.1007/BF03222789

[26] Stolyarova D. Y., Kuznetsov N. M., Belousov S. I., Chvalun S. N.: Electrorheological effect in low filled suspensions of highly anisometric montmorillonite particles. Applied Clay Science, in press (2018).

[27] Kuznetsov N. M., Shevchenko V. G., Stolyarova D. Y., Ozerin S. A., Belousov S. I., Chvalun S. N.: Dielectric properties of modified montmorillonites suspensions in polydimethylsiloxane. Journal of Applied Polymer Science, 135, 46614/1-46614/9 (2018).

https://doi.org/10.1002/app.46614 